\title{
Application of High-Frequency Gravitational Waves to the Cataclysmic Event of Our First Encounter with Intelligent Extraterrestrial Beings
}

\author{
Robert M. L. Baker*, Bonnie Sue Baker \\ GravWave Division, Transportation Sciences Corporation, Palm Desert, CA, USA \\ Email: "DrRobertBaker@GravWave.com
}

Received 19 November 2015; accepted 23 January 2016; published 26 January 2016

Copyright (C) 2016 by authors and Scientific Research Publishing Inc.

This work is licensed under the Creative Commons Attribution International License (CC BY). http://creativecommons.org/licenses/by/4.0/

(c) (i) Open Access

\begin{abstract}
Three advances are proposed as a pathway to the cataclysmic event of our first encounter with intelligent extraterrestrial beings. First, discovery of very large numbers of extraterrestrial planets, "exoplanets" (possibly as many as10 ${ }^{23}$ in our Universe); second, introduction of electronic components into the human body evolving into a cybernetic and biological "cyborg," a model for an extraterrestrial being Cyborgs might allow advanced civilizations to endure hundreds of thousands of years. Third, the recent development of high-frequency gravitational wave (HFGW) detectors, the communication means of choice for an advanced cyborg civilization since they are not easily absorbed like electromagnetic radiation. Six HFGW detectors are presented for application to our first encounter with intelligent extraterrestrial beings. Numerical estimates are made for the failure of extraterrestrial civilizations such that no two exist at the same time (Fermi's Paradox). It is concluded that there might remain at least $\approx 1.48 \times 10^{8}$ Worlds intercommunicating with HFGWs at any one time in any one region of our Universe. The predicted form of extraterrestrial beings is by means of animaginary, but based upon comprehensively documented and detailed projection of the evolution of "Earthling" homosapiens, to become "cyborgs." It is proposed that such long-living cyborg forms of intelligent beings would be encountered by us. The first cataclysmic encounter with them is expected to be interception of their interstellar communications. The predicted frequency of intercepted messages under one set of assumptions is at least $\mathbf{1 5 0 0}$ per day. After decoding the intercepted messages, keys may be found to improve vastly the present and future quality of life for us earthlings. Advanced beings might utilize direct brain-to-brain communication and it is concluded that research into brain-to-brain communication and HFGW detection are encouraged.
\end{abstract}

\footnotetext{
*Corresponding author.
}

How to cite this paper: Baker, R.M.L. and Baker, B.S. (2016) Application of High-Frequency Gravitational Waves to the Cataclysmic Event of Our First Encounter with Intelligent Extraterrestrial Beings. Journal of Applied Mathematics and Physics, 4, 110-129. http://dx.doi.org/10.4236/jamp.2016.41015 


\title{
Keywords
}

\author{
High-Frequency Gravitational Waves, Search for Extraterrestrial Intelligence, Gravitational Waves, \\ Exoplanets, Cyborg
}

\section{Introduction}

In the past several years, there have been dramatic advances in at least three areas of technology that suggests a rather immediate pathway to the cataclysmic event of our first encounter with intelligent extraterrestrial beings. The most dramatic advance has been the observational results of the Kepler satellite observatory. These observations not only verified the existence of several exoplanets, but led to the discovery of the previously unpredicted extreemly large number of them; as would be calculated, possibly $10^{23}$ of them in our Universe. The second advance has been taking shape over the past several decades, but most dramatically over the last few years. Broadly speaking, this advance is the introduction of electronic components into the human body and especially into the brain. The clear outcome would be human evolution to a combination cybernetic or electronic and biological human being: a "cyborg." It was reported by Robert F. Service [1] in the Journal Science "The beginnings of a cyborg world have already arrived ...!" and Manuela Veloso who suggested that we "embrace a robot-humanworld" [2]. It is proposed that such cyborg forms of intelligent beings would be encountered by us and would alllow advanced civilizations to endure hundreds of thousands of years. Civilization's endurance is key to estimating the possibility of such encounters with extraterrestrial beings. Therefore, given such long endurance, a large number of such intercommunicating Worlds would be active at any given time and interception of their messages quite possible. The third dramatic technical advancement began in about 1990 to 2000 when Mike Cruise [3] designed an electromagnetic detector for very-high-frequency gravitational waves at Birmingham University, England. Since then, some five additional high-frequency gravitational wave (HFGW) detectors have been either fabricated or designed. Among the latest is the Stanford/University of Nevada 50 to $300 \mathrm{kHz}$ HFGW Detector [4]. HFGWs present the optimal communication means for an advanced, cyborg, extraterrestrial civilization to utilize both for their interstellar and for their local, on-planet communications. An appropriate HFGW transmitter for such communication is discussed by R. M L., Jr. and B. S. Baker [5]. HFGWs are not absorbed by matter as are electromagnetic waves. Furthermore, it is suggestred that the cataclysmic event of our first encounter with intelligent extraterrestrial beings will be based upon the interception of such unabsorbed or unattenuated HFGW messages.

Is it correct that our first encounter with intelligent extraterrestrial beings will be a cataclysmic event rather than another monumental scientific achievement? If so, then it would behoove us to become emotionally prepared. Earthlings seem to have need for a superior, if not supreme, being. Our earthling need may arise from a psychological feeling that we need not take responsibility for our own actions and instead allow some deity to take responsibility. Alternatively, it may arise from some primal belief that there are vitally important signals from fire, water, volcanoes, from the stars above to guide us. This need even relates to the definition of "consciousness." As Allison Abbott [6] suggests, "Where does consciousness arise? In the soggy grey mass of the brain, as materialists would have it? Or 'elsewhere'-assigned down the ages variously to God or the cosmos ..." Perhaps, an extraterrestrial intelligence would have the answer. On the other hand, some of those who assume a Supreme Being believe consciousness to be a sacred gift and the rational scientific mind and resulting physical laws, as faithful servants. It is difficult for them to obtain a framework for discussing the conjectured form, evolution and intercommunication activity of possibly more than a billion, billion of advance intelligent beings now alive in billions of other worlds. Many of these concepts are discussed in David Wilkinson's book [7]. There is even a belief that interception of interstellar communication among advanced exoplanetary beings should be avoided since they might upset their religious or spiritual "balance.” Tyco Brahe’s observations, which led Kepler to heliocentric theory, were greatly resisted by the Church, like Copernicus before them. Some of us feel that our lives are programmed in advance, "fatalistic," that we have no absolute control of our actions or destiny — only some superior being or "force" ultimately controls us. Others of us believe that there exists some "POWER" that guides us, a power who supports good behavior and punishes bad behavior—a God, Allah, and, the opposite, a Satan; who are both supernatural and all powerful. Super people, who are immortal and may 
guide us (if we are worthy) into immortality—into a life beyond death—for example, to an underground chamber in a pyramid where we may become immortal! Lizzie Wade [8] stated "Although much of Egyptian cosmology is alien today, some is strikingly familiar: The gods of today's major religions are also moralizing gods, who encourage virtue and punish selfish and cruel people after death.” Earthlings seem to yearn for such philosophies or "religions" and truly, we yearn for "something" far beyond our selves. So our first encounter with an extrasolar intelligent life form will not simply be the most fantastic scientific event encountered in the history of Man—no, it will be a cataclysmic event beyond all imagination! Would the first validated interception of an interstellar message be of immediate, practical value to humanity? Probably not. Nevertheless, after decoding many of these interstellar messages, keys may be found to vastly improve the present and future quality of life for us earthlings.

\section{Exoplanets}

61 cygni is the first star other than the Sun to have its distance from Earth measured. The star's large angular motion relative to the other stars (termed proper motion) allowed for triangulation or surveying to determine its distance. In the 1960s, it was thought that this nearby star (about nine light years away) had a planet on orbit around it—but it was later proved planetless. Confirmation of such "exoplanets", came in the early 1990s, when astronomers found compelling signs of smaller bodies orbiting stars. These signs included the wobble of a star caused by orbiting planets and eclipses caused by a star's transiting planet as measured, thousands of stars at a time, by the plant-hunting Kepler satellite observatory. In November 2001 came the first observation of an exoplanet atmosphere (HD 209458 b). As of 2013 Cruz and Coontz [9] estimated that our Galaxy (and there are 100 to 200 billion galaxies in our Universe) contains at least as many planets as stars and there are 200 to 500 billion stars per galaxy! In addition, according to Brandeker [10] there is “... at least one planet for every solartype star.” Thus, there may be as many as about $(200,000,000,000) \times(500,000,000,000)=$ $100,000,000,000,000,000,000,000\left(=10^{23}\right.$ or one followed by 23 zeros or one hundred sextillion) exoplanets out there! In the four years after its launch, Kepler had monitored the brightness of some 150,000 stars and it has discovered more than 2700 possible exoplanets. By 2013, follow-up observations by ground-based telescopes had confirmed 122 exoplanets. Roughly, half of these candidates are estimated to be twice the size of the Earth or smaller such as a Mars-sized planet [11]—many of these could be rocky planets. The findings suggest that hordes of earthlike planets exist in the habitable zones of stars and are waiting to be discovered! In today's findings the habitable zone (sometimes referred to as "Goldilocks Zone") of a star is defined as "the annulus around the star, where a rocky planet with a carbon dioxide water and Nitrogen atmosphere, has sufficiently large water content (such as the Earth) so that it can host liquid water on its surface.” Thus the temperature would need to be between the freezing point and the boiling point of water [12]. However, the habitability zone must be considered on a case-by-case basis rather than that one-size-fits-all definition using water as the only possibility. Also there might be Silicon-based rather than Carbon-based living creatures (Silicon and Carbon have similar chemical properties), might exist in a Hydrogen-rich rather than an oxygen-rich atmosphere and other qualities that might support the development of intelligent beings. Any possible lifeform should not be ruled out. In fact, consciousness or intelligence might exist within stars or within any structure, even within dark matter in the Universe! As Caleb Scharf [13] states: "Who is to say that life must consist of discrete, self-replicating organisms that move around independently, competing, cooperating, and mating with one another?” Nevertheless, for such "intelligence" to matter the ability to communicate is essential. Every month, the orbiting Kepler satellite telescope alone observes hundreds of new potential habitable exoplanets in a patch of sky near the constellation Cygnus. Just in July, 2014, the exoplanet Kepler-93b was discovered having a planetary radius 1.48 that of the Earth and a planetary mass roughly 50\% larger than Earth [14] and exoplanet GJ1132b was discovered to even more closely resemble Earth [15]. GJ1132b may be the most Earth-like exoplanet found at this writing (2015). It is located in the habitable zone of a M-dwarf star, about 39 light years away, is about $20 \%$ larger than our own planet and has a density consistent with an Earth-like planet [16].

However, the Kepler satellite is not the only planet hunter. Recently on two mountaintops in Chile, two stateof-the-art instruments start scanning the skies for planets around other stars next year [17]. The vast majority of the over 1000 exoplanets identified so far have been found using indirect methods because the starlight wipes out their faint optical signals. However, the new instruments, one North American and one European, will see planets directly! Fixed to two of the world's biggest telescopes, they push optical technology to the limit. "After 
10 years building it, to see it view the sky is fantastic,” says Bruce Macintosh of the Lawrence Livermore National Laboratory in California, principal investigator for the planet imager. A consortium of US and Canadian institutions has built the telescopes. They report on their Planet Hunters website and have found a new exoplanet: PH3c; which has a substantial atmosphere of hydrogen and helium gas. (www.planethunters.org). As reported by Witz [18] "By the mid-2020s budgets permitting, astronomers expect to have a satellite called the Wide-Field Infrared Survey Telescope (WFIRST) busy cataloging planets that are too far away from their host stars for Kepler to have spotted them" and the "European Space Agency is planning the Planetary Transits and Oscillations of Stars (PLATO) a 2024 exoplanet mission ..." as well as NASA's Transiting Exoplanet Survey Satellite scheduled for 2017.

\section{The Form and Longevity of an Extraterrestrial Intelligent Being}

Those 100 sextillion or 100,000,000,000,000,000,000,000 exoplanets come into existence quite naturally in most star and multiple-star systems. However, how about the creation of "life" on some of those exoplanets that are in the habitable zone [19] or rather in a zone that is presumed to be a habitable zone of their star (or double star [20]? There is a high probability that there exist huge numbers of potential "Cradles of Extraterrestrial Intelligent Beings" out there. However, how will we encounter them and what will we do when we do encounter them?

So far, scientists have been unableactually to "create life from scratch" as it were. Deamer [21] suggests that deep-sea hydrothermal may have provided the condition for the origins of life-the "first spark". Another possible "spark" was suggested by Sid Perkins [22] that from some Czech laser evidence "... that the hellish conditions produced when an asteroid or comet slams into Earth could have created some key building blocks of life on Earth.” Knoll also discusses the origin and early evolution of life in his book [23]. That is not the crucial question. What we really would like to know is how life forms evolve on these far Worlds and even more importantly what would be their form and life span for those that might be far more advanced than we earthlings. As Suzan Wolf's review [24] of Edward Wilson's book The Meaning of Human Existence [25] suggested “...we are apt to be similar to any possible extraterrestrial species with level of intellect comparable ... to ours." Since we have only one example of an intelligent life form, our own, we can only project how our humankind might evolve. Like Wilson's conjecture, we then could at least guess that advanced intelligent civilizations on one or more exoplanets might have a similar evolution as on Earth. It seems likely, as will be discussed, earthlings will probably evolve into a blend of biological and electronic creatures, that is into cyborgs!

But is this the way in which humanity and, by extension, alien entities will evolve? Christof Koch [26] in his review of Kurzweil's book: "How to create a Mind" [27] states, "Science-fiction novels and films have long popularized the notion that machines will, sooner or later, match and ultimately exceed human-level intelligence. On the way they will acquire feelings and coconsciousness. In the movie Blade Runner, a replicant exclaims in the face of his imminent demise 'I've seen things you people wouldn't believe ...'”. Koch [26] further pooh poohs the idea that "...that biological limitations, including aging and insufficient memory and intelligence, should, and will be transcended by nanotechnology and artificial intelligence (AI)." (Termed "Transhumanism”) Koch insists that it will never be a pure machine it will be-a combination or blend of biological and electronic elements acting together; essentially the cyborg that Robert Service suggests [1]. The biological elements will allow for art, invention, humor, emotion, fun, etc. and the electronic elements will provide enhanced functions such as memory, logic, physical capability, computation, optimum decision making (Gershman, [28]), etc.

There are fictional projections of homo sapien's evolution. Nevertheless, research progress is real, as a mix of biologist, materials scientist, and nanotechnology experts are chipping away at a host of challenges. "I see it as building a seamless interface between cells, tissues, and electronics," says Aleksander Noy, a bio-nano-electronics expert at Lawrence Livermore National Laboratory and the University of California, quoted by Service [1]. For now, most of these efforts focused on providing better health care and quality of life for patients. Nevertheless, over time, expect devices “... that will make us better athletes and soldiers," or even reduce our facial wrinkles! For example the Electrolift ${ }^{\circledR}$ mask device discussed by Baker [29]. "A few years ago these things were science-fiction. But now we are seeing the emergence of real devices and applications," Noy [30]. "And fast, ...” says ZhenanBao, an organic electronics expert at Stanford University in California: "The competition is furious" according to Service [1]. The idea of fusing man and machine has long tantalized humanity. Over the past century, researchers have pioneered myriad efforts to use electronics to measure biological activity and sometimes 
even alter it. According to Underwood [31], Geoffrey Ling, atop biotechnology research official at the Defense Advanced Project Agency, challenged neuroscientists to do something extraordinary: "Develop an implantable device in a human brain that can reverse memory loss. Also UCLA's Brain Institute and other universities are developing electronic prostheses that interact with brain regions critical to memory. Victims of stroke may soon have improvement due to brain implants that still allow individual decision making or "thinking" (Ganguly, [32]). Michael McAlpine, a mechanical engineer at Princeton University, and colleagues (Machens, [33]) reported in the May 1, 2013 issue of NanoLetters (Mannoor, [34]) that they've made the first 3D printed functional organ: a bionic ear that, shown in Figure 1, "hears" acoustic sounds and ultrasounds. "We're trying to see if one could introduce augmented functionality that a human wouldn't ordinarily have..." McAlpine says. "Organs from the lab ..." appear to be realistic even today as discussed in the June 18, 2015 issue of Nature (Marx, [35]).

Some three-dimensional (3D) printers work by using computer-driven laser printer to build up layers of material based inks, usually made from plastic. One team (Ledford, [36]) started with three different inks: one made from silicone; another with silicone infused with silver nano-particles; and a third with chondrocytes, cells that produce cartilage, along with the gel to promote their growth. Dongsheng Liu [37] at Tsinghua University in Beijing, Wenmiao Shu at Heriot-Watt University in Edinburgh, UK, and their team. Lui, made two water-based inks from peptides and synthetic DNA strands that form a stable hydrogel when mixed. Numerous groups have used 3-D printing to make tissues, but they have typically printed only scaffolding materials and cells. The first team added a level of sophistication to the technology. The researchers printed out a metal coil in the center of an engineered ear that serves as an antenna capable of picking up acoustical signals and converting them into electrical pulses for the inner ear à la a conventional cochlear implant. Attempts to develop electronic organ function led to the development of bioelectric batteries (Fritzsch, [38]). Artificial electronic organs will function with organic batteries and drive implanted devices such as cochlear implants or pacemakers (Hanson, [39]). It might even be possible today to turn nonfunctional muscles into electronic organs, thus generating enough bioelectric energy to drive small electric motors in already existing exoskeletons for human performance augmentation (Kazerooni, [40]). Then there is the "Brain Chip". A computer called "True North" accomplishes pattern recognition--a major function of our brain. True Northcontains 5.4 billion transistors wired together to form an array of 1 billion digital "neurons" that talk to each other via 256 million "synapses" the intercommunication media of the brain.” It gets almost everything right," says Dharmendra Modha, an electrical and computer science engineer who leads the artificial brain project at IBM's Almaden Research Center in the hills beyond Silicon Valley (Merolla [41]).

Finally, there is the brain-machine interface or BMI. Back in 2011 researchers described a prosthetic system that provides tactile feedback by stimulating the somatosensory cortex, the brain region responsible for the perception of touch). Andrew Schwartz at the University of Pittsburg in Pennsylvania and his colleagues implanted two microelectrodes in a woman's motor cortex, the part of the brain responsible for initiating movement. The devices recorded cortical activity associated with her desire to move a robotic arm. An external decoder connected to the electrodes and the arm converted these signals into electronic instructions for the artificial limb. As shown in Figure 2(a) and Figure 2(b), within 13 weeks she could grasp and move objects almost as fast, smoothly and accurately as an able-bodied person! (Reardon, [42]).

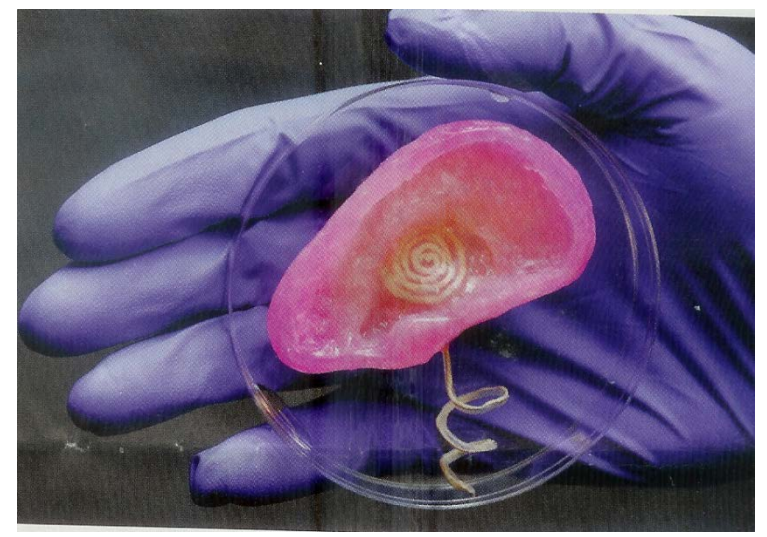

Figure1. Engineering a Functional Ear (Service, [1], Mannoor, [34]). 


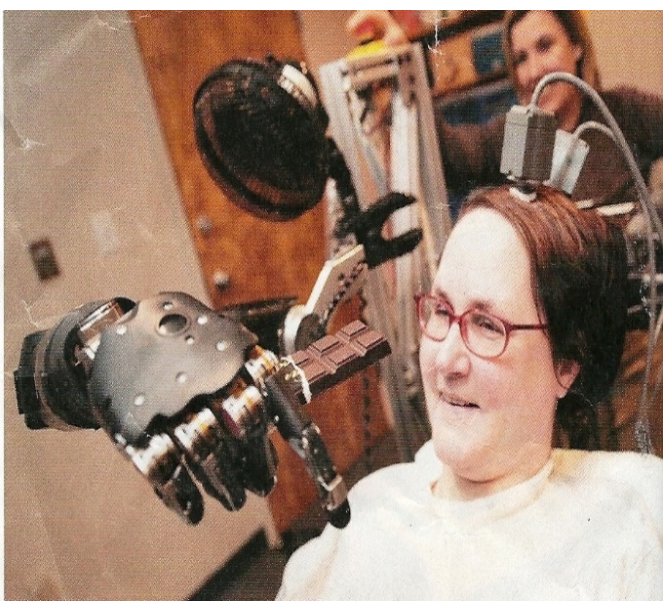

(a)



(b)

Figure 2. (a) Brain-controlled mechanical arm and hand (Reardon, [42]), (b) "imagine that!” a brain-controlled mechanical hand (Pruszynski and Diedrichsen, [43]).

If advances humankind evolves into a cyborg entity, then it will be due to the aforementioned documented technological; most of which have already been made. Furthermore, such entities could well be repairable and include technological improvements in their "parts" as time goes by. They will evolve into ever improving creatures somewhat like the voluminous cathode ray, Black \& White TVs of the 1950s evolved into the color, flat-screen TVs of today. Therefore cyborgs could be immortal and constantly improving - that is until, to be discussed, they may somehow eliminate themselves! In any event, it is concluded that the human lifespan on our Earth will dramatically increase with the advent of the aforementioned advances in "cyborg technology" and the duration of an Extraterrestrial-Intelligent-Being civilization could be in the hundreds of thousand years. Obviously, such an advance would produce a myriad of new problems for humanity such as the necessity for stringent population/birth control, new governmental paradigms-not only transition to a huge aging population but possibly an "ageless" population!

\section{How Soon Will We Discover Alien Life?}

Based upon the Kepler Satellite Observatory data there are approximately as many exoplanets as stars (Cruz, [9] and Brandeker, [10]). That does not mean that there is an exoplanet around every star , but rather that, for example, for every 8 stars there may be one star with 8 planets around it, like our Sun, or two of these stars with 4 exoplanets around each or one with 5orbiting exoplanets and one with 3 , etc. It is important not only to predict how many exoplanets are around us and how these cyborg-stage, interstellar-communicating, civilized-entities may mutually function, but also to reexamine how many "life-supporting" exoplanets are out there. Although these "Cyborg Civilizations" may be composed of immortal entities, they may also exhibit a "mean time to failure"! That is they may destroy themselves: self-destruct or have conflicts like the "Star Wars" sagas and obliterate each other or be destroyed like the dinosaur extinction such as discussed by Erwin [44]. This is the so-called Enrico Fermi paradox in which civilizations "blink" on and off and there may never be two advanced civilizations "operating" or "intercommunicating" at any given time. Fermi reasoned that this would explain why Earth may never be visited by an advanced exoplanet civilization - there would never be "UFOs"! Let us pursue this last point in more detail. Consider the time line or string of Figure 3.

Point $\mathbf{A}$ is the beginning of our Universe about 13.7 billion ago; point $\mathbf{B}$ is the beginning or birth of an exoplanet's first "spark" of life. B to TODAY (1) includes the approximately 11,700 year-long Holocene period for earthlings. Point $\mathbf{C}$ is the start of the advanced or cyborg civilization stage during which an advance civilization will, according to this theory, utilize interstellar communication with other advanced civilizations that may be intercepted by earthlings. Let us introduce some arbitrary numbers-all very speculative. Suppose that half of all exoplanet civilizations "started", their $\mathbf{B}, 8$ billion years ago or some $13.7-8=5.7$ billion years after $\mathbf{A}$ and had already reached $\mathbf{D}$ (demise). That is, let it be assumed that we should consider only half of the exoplanet-bearing stars in our vicinity of the Universe as possible candidates for interstellar communicate; the rest having already 


\begin{tabular}{|c|c|c|c|c|c|}
\hline TODAY (1) & TODAY (2) & TODAY (3) & & & \\
\hline$\underline{\mathbf{A}}$ & B & C & $\uparrow$ & D & $\uparrow \rightarrow$ TIME \\
\hline
\end{tabular}

Figure 3. Exoplanet advanced civilization time line or string.

met their demise. Various archeological estimates show that the "spark of life," on Earth started about 3.6 billion years ago. If we earthlings are now on the verge of the cyborg, interstellar-communication interception phase, at $\mathbf{C}$, then the time interval between $\mathbf{B}$ and $\mathbf{C}$ about is about 3.6 billion years, based upon very speculative estimates of the explosive increase in longevity of earthlings due to the rapid evolution to a biological/electronic or cyborg life form and the inherent desire for a long productive lifetime (resulting from repairable and/or replaceable "parts"). It is estimated that there would be lifetimes of several thousand years and thousand-yearlong generations. Of course, our major interest is in the interstellar communication and/or cyborg phase of and exoplanet civilization's life that is between $\mathbf{C}$ and $\mathbf{D}$. Consider this interval's time period in terms of the number of a civilization's generations (single step in the line of descent from an ancestor and the usual period of a society's evolutionary steps) prior to its demise. For example, if demise occurs after 400 of the 1000 year-long generations or $400 \times 1000=400,000$ years, then this would be the window of opportunity for these advanced civilizations to accomplish interstellar communication. Please see Table 1 for other numbers of generations and their lengths in years utilized to determine the time between $\mathbf{C}$ and $\mathbf{D}$. As a point of reference after emerging in Africa about 60,000 years ago, our lifetimes have averaged from about 30 to 75 years and generations have varied very roughly from 15 to 25 years in length. Thus up to $\mathbf{C}$ our civilization would have survived about 2000 to 4000 generations. Assuming our civilization is about to evolve rapidly into advanced cyborgs at Cit is estimated (or "conjectured")the longevity of our and other civilization near us in our Galaxy to average about 400,000 years C to D. Various other alternatives are exhibited in Table 1.

However, the demise of the advanced civilization might occur almost any time during the time interval between $\mathbf{B}$ and $\mathbf{D}$. That is, during that time interval between $\mathbf{B}$ and $\mathbf{D}$ advanced civilizations could "blink" on at $\mathbf{B}$ and then off, that is reach their $\mathbf{D}$. There are numerous values that could be chosen for these dates. Let us speculate that civilizations reach their $\mathbf{D}$ in a serial fashion. Of course, there could be overlap and the time spans would be quite randomly distributed. In order, however, to get some approximate numerical results simply assume that when one advanced civilization reaches their $\mathbf{D}$ (blinks off) another civilization reaches their $\mathbf{C}$ (blinks on). In the numerical example the number of such intervals would be 3.6 billion years divided by 400,000 years or 9000. By the way, only if TODAY were at TODAY (2) would a particular one of the 9000 advanced civilizations have the opportunity for interstellar communication that could be intercepted by us. But only those Worlds that are clustered together close enough to communicate with each other in a time span less than 400,000 light years apart in the numerical example could communicate in time before their demise $\mathbf{D}$. Let us suppose that the average distance apart of stars in our Galaxy is about five light years (see, for example,

http://boojum.as.arizona.edu/ jill/EPO/Stars/galaxy.html), so that minimum back and forth intercommunication time would average some $2 \times 5=10$ years for this pair of close by stellar exoplanetary systems. Nevertheless, during their advanced phase of 400,000 years (C to D) a given advanced civilization could intercommunicate with other advanced civilizations that were within at most 200,000 light years (communiqué transmitted with the speed of light at the beginning of their advanced cyborg stage $\mathbf{C}$ and the reply received just before their demise D). That is they could communicate with any advanced interstellar communicating civilization within 200,000 light years. Since there are about five light years distances on average between stars (and their assumed exoplanet companions) there would be an intercommunications "range" of 200,000/5 $=40,000$ exoplanetary systems in any direction (termed S for "star distances"). Specifically, we could send a message off (at the speed of light) at the dawn of the cyborg (interstellar communication) stage and have it received by an advanced civilized exoplanet at a 200,000 light year distance in 200,000 years. If they made an immediate reply, then the sending civilization would receive a reply from the advanced interstellar communicating civilized exoplanet within another 200,000 years just before the sender's demise. So if, on average, they are five light years apart, there would be some forty thousand intercommunicating exoplanets in any given direction, so the spherical volume around the sender would contain about, $(4 / 3 \pi)(40,000)^{3}=2.7 \times 10^{13}$, such exoplanetary systems to intercommunicate and for us to eavesdrop on. In general, if we define $d$ as the time interval $\mathbf{C}$ to $\mathbf{D}$ in years as found, for example, in the entries of Table 1, then given a 5 Light Year average distance apart of the stars and their advanced exoplanet civilizations in our neighborhood of the Universe, they could have the possibility of one back-and-forth communication with another advanced civilization as far as $S=(d / 5) / 2=d / 10$ stars away. In thenumerical example $S=$ 
Table 1. The length of time in years between a civilizations' emergence of cyborgs and/or interstellar communication capability and their demise, $\mathbf{C}$ to $\mathbf{D}$ or $d$.

\begin{tabular}{|c|c|c|c|c|c|c|}
\hline $\begin{array}{c}\text { No. of generations to } \\
\text { D } \rightarrow \text { generation length, years } \downarrow\end{array}$ & 1 & 4 & 40 & 400 & 4,000 & 40,000 \\
\hline 25 & 25 & 100 & 1000 & 10,000 & 100,000 & $1,000,000$ \\
\hline 100 & 100 & 400 & 4000 & 40,000 & 400,000 & $4,000,000$ \\
\hline 1000 & 1000 & 4000 & 40,000 & $400,000^{\mathrm{a}}$ & $4,000,000$ & $40,000,000$ \\
\hline 10,000 & 10,000 & 40,000 & 400,000 & $4,000,000$ & $40,000,000$ & $4 \times 10^{8}$ \\
\hline 100,000 & 100,000 & 400,000 & $4,000,000$ & $40,000,000$ & $400,000,000$ & $4 \times 10^{9}$ \\
\hline $1,000,000$ & $1,000,000$ & $4,000,000$ & $40,000,000$ & $400,000,000$ & $4,000,000,000$ & $4 \times 10^{10}$ \\
\hline
\end{tabular}

${ }^{\mathrm{a}}$ The numerical-example value.

$400,000 / 10=40,000$. Thus the number, $n$, of such potentially intercommunicating civilizations in the spherical volume of interstellar communicating exoplanet civilizations would be

$$
n=(4 \pi / 3) S^{3}
$$

which is somewhat similar to the factor $R_{*} \times f_{p}$ in the Drake equation, http://en.wikipedia.org/wiki/Drake.

In the example, $n=2.68 \times 10^{14}$. Of course, this number must be greatly reduced, which also will reduce the estimated number of the true potential intercommunicating advanced civilizations around Earth. Let us assume that only one out of ten Exoplanets would be in the habitable zone between the freezing and boiling point of water (probably conservative because not all intelligent extraterrestrial life may need to be in this temperature range). Next, let us assume that only one out of ten of these habitable Exoplanets will reach the advanced stage C. Finally, cut their number in half to account for very old and dead Exoplanetary civilizations and then by dividing by the number of stars that have reached $\mathbf{C}$ but have not reached $\mathbf{D}$ or, in the numerical example, divided by $2 \times 9000$. So, under these arbitrarily parameters, the actual number $N$ of potential interstellar-communicating Exoplanets is estimated to be

$$
N=2.68 \times 10^{14} / 10 \times 10 \times 2 \times 9000=1.485 \times 10^{8}
$$

In general, the series of "operating" civilizations that blink on and off in a series during the 3.6 billion year time span, discussed above, that is $2 \times 3.6 \times 10^{9} / d$, so that Equation (1) becomes

$$
N=(4 \pi / 3) S^{3} d / 200 \times 3.6 \times 10^{9}=(4 \pi / 3)[(d / 5) / 2]^{3} d / 7.2 \times 10^{11}=5.8 \times 10^{-15} d^{4}
$$

Therefore, in the example $N=1.485 \times 10^{8}$ as before where $N=$ the estimated number of the true potential intercommunicating advanced civilizations around Earth at any particular time. (Of course, one would only be interested in our Sun and Earth being in the center of the intercommunicating group or volume for eavesdropping not on the surface or boundary.) In Table 2 is to be found values of $N$ as a function of $d$. Figure 4 exhibits an example of the "communication sphere's" boundary surface. It is to be emphasized that each intercommunicating advanced civilization may be comprised of thousands if not millions of independent interstellar transmitting/receiving individuals or cyborg entities. There are about three million independent radio operators or "hams" worldwide on our planet (Silver, [45]). Alternatively, an advanced civilization might evolve into a single-entity cyborg, but perhaps with billions of input/output communications ports. Thus, the $N$ represents the very minimum of potential intercommunication links.

As a further example, if, on the other hand, the sending exoplanet attempted a intercommunication with an exoplanet 40,001 exoplanets distances or 200,005 light years away when it just reached the $\mathbf{C}$ stage, then the reply message reach the sending exoplanet in 200,010 years (similar to the red communication links in Figure 4)— too late since the receiving exoplanet had already reached its demise, $\boldsymbol{D}$, so no intercommunication! In any eventthere would not be much of a conversation with 200,000 light-year distant exoplanet. If, however, at a lesser distant exoplanet a slightly more relaxed chat with very long pauses-actually like an exchange of letters. There would be two complete sets of send/receive messages if exoplanets 100,000 light years apart. In this case there would be about $(1 / 2)^{3}$ or one eighth the total number of exoplanets (eighteen million) in the sphere or about 1,875,000 possible exoplanet communication partners to be overheard. Of course, the communication would be interstellar correspondence not conversation! Let us pursue the value of close interstellar communica- 


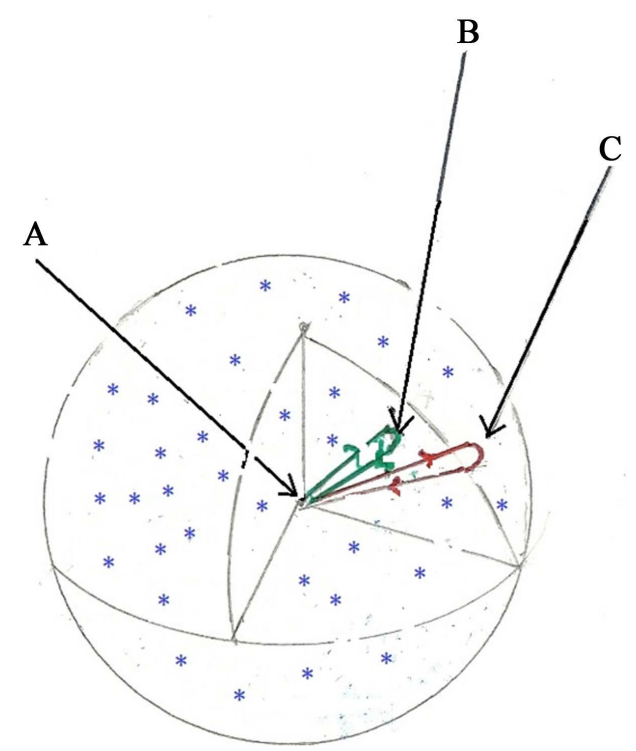

Figure 4. The boundary sphere of radius 5000 light years containing stars in the neighborhood of our Sun whose advanced civilizations could intercommunicate. (A) Message sent by an advanced exoplanet civilization near (less than about 100 light years) our Solar System (to a 5000 light-year distant exoplanet) just at a time when the civilization reached stage $\mathbf{C}$ and a reply received from that exoplanet 5000 light years away just in time $(10,000$ years after it was sent) before the advanced civilization blinked off at D. Another message sent by that advanced exoplanet civilization near our Solar System to a 5005 light-year distant Exoplanet just at a time when that advance civilization reach stage C, but "Oh, Oh!” it had just blinked off at $\mathbf{D}$ after it had endured 10,000 years, or ten years before the message reply was at 10,010 years since transmission-thus the transmission failed and interstellar intercommunication could not have been achieved. Therefore only advanced exoplanet civilizations 5000 light years away or less can be communication partners with advanced exoplanet civilizations if they all have 10,000 year $\mathbf{C}$ to $\mathbf{D}$ intervals and are synchronized as to "start times" $\mathbf{C}$. These assumptions are meant to convey the concept and the results of this example are, therefore, very approximate; (B) Advanced exoplanet civilization here receiving a message from another advanced exoplanet civilization (green communications path), some 5000 light years away, whichwas sent 5000 years ago by this advanced civilization exoplanet and immediately replied to! (C) An advanced exoplanet civilization here receiving a message from another advanced exoplanet civilization (red communications path in Figure 4), some 5005 light years away, which was sent 5005 years ago by this advanced exoplanet civilization and immediately replied to!

Table 2. Number, $N$, of potential intercommunicating advanced civilizations as a function of the years between $\mathbf{C}$ to $\mathbf{D}$ or $d$.

\begin{tabular}{cc}
$d$ years between $\mathbf{C}$ to $\mathbf{D}$ & $\mathrm{N}$ \\
\hline 4000 & 1.48 \\
40,000 & 14,800 \\
$\mathbf{4 0 0 , 0 0 0}$ & $\mathbf{1 . 4 8} \times \mathbf{1 0}^{\mathbf{8 a}}$ \\
$4,000,000$ & $1.48 \times 10^{12}$ \\
$40,000,000$ & $1.48 \times 10^{16}$ \\
\hline
\end{tabular}

\footnotetext{
${ }^{a}$ The numerical-example value.
}

tion partners. It clearly is not the sheer number of interstellar communication partners that counts, but the frequency of their messages that increases the probability of message intercept. Although a reduction in $d$ greatly reduces the number of advanced civilizations that might be intercommunicating, it also allows for more frequent messaging. In particular if $d$ is reduced by a factor of ten there would be $1 / 1000^{\text {th }}$ the number of available stars and their companion advance civilization or $N_{1}=N / 1000$. On the other hand, the ability to have some five back and forth communication during their joint lifetimes is achieved. Not only five but many more, say 25 or $N^{2}$ times more once intercommunications was accomplished after a dialog initiated! In Table 2, $d$ is utilized as the parameter and for each $d$ we compute another $N_{1}$ for $0.1 d\left(N_{1}=N / 1000\right)$, yet another $N_{2}$ for $0.001 d\left(N_{2}=N / 10^{6}\right)$ 
and $\left(N_{1}\right)^{2}$ and $\left(N_{2}\right)^{2}$ for an estimate of the number of actual messages that might be transmitted and/or received for $0.1 d$ and $0.001 d$. In the numerical example $N_{1}=1.48 \times 10^{5}$ and $N_{2}=148$ so that the total number of messages received over the $0.1 d$ and the $0.001 d$ are estimated to be $N_{1}^{2}=2.2 \times 10^{10}$ and $N_{2}^{2}=22,000$ respectively. Next compute the frequency in messages per year that might be intercepted by a listening post on our Earth. Under the foregoing assumptions those message frequencies are given by

$$
f_{1}=\left(N_{1}\right)^{2} / 0.1 d \text { per year }
$$

and

$$
f_{2}=\left(N_{2}\right)^{2} / 0.001 d \text { per year. }
$$

So that $f_{1}=550,000$ per year and $f_{2}=55$ per year. They are presented in the last two columns of Table 3 . In the numerical example for the $0.1 d$ case, there might be 550,000 possible messages to intercept each year or about 1500 per day.

It is also interesting to note the distance of the stars/exoplanets in light years for $0.1 d$ and $0.001 d$. These distances are exhibited in Table 4 and, for the most part, are in our Galaxy.

Not all of these exoplanetary systems in the sphere of Figure 6 would have planets in the habitable zone and not all will become cradles of intelligent life forms that evolve into the cyborg stage. Here again it is conjectured that one out of one hundred will arise as advanced civilizations that development interstellar communications.

Additionally, it is not known exactly how many will be "blinked on" at the same time according to Fermi's Paradox and therefore able to be participants in an interstellar intercommunications network. As has been calculated in the numerical example, their potential interstellar intercommunications network will contain about $1.4 \times$ $10^{8}$ members or possible Worlds. Furthermore, like we earthlings there will also exist "Explorer groups" sent-

\begin{tabular}{|c|c|c|c|c|}
\hline $\begin{array}{c}d \\
\text { Years between } \mathbf{C} \text { to } \mathbf{D}\end{array}$ & $\begin{array}{c}\left(N_{1}\right)^{2} \\
\text { Total messages over } 0.1 d\end{array}$ & $\begin{array}{c}\left(N_{2}\right)^{2} \\
\text { Total messages over } 0.001 d\end{array}$ & $\begin{array}{c}f_{1} \\
\text { Messages per year }\end{array}$ & $\begin{array}{c}f_{2} \\
\text { Messages per year }\end{array}$ \\
\hline 4000 & $\approx 0$ & $\approx 0$ & $\approx 0$ & $\approx 0$ \\
\hline 40,000 & 222 & $\approx 0$ & $\approx 0$ & $\approx 0$ \\
\hline 200,000 & $8.66 \times 10^{7}$ & 86.6 & 4,330 & 0.43 \\
\hline 300,000 & $2.2 \times 10^{9}$ & 2,200 & 74,000 & 7.4 \\
\hline $400,000^{a}$ & $2.2 \times 10^{10}$ & 22,000 & 550,000 & 55 \\
\hline $4,000,000$ & $2.2 \times 10^{18}$ & $2.2 \times 10^{12}$ & $5.5 \times 10^{12}$ & $5.5 \times 10^{8}$ \\
\hline $40,000,000$ & $2.2 \times 10^{26}$ & $2.2 \times 10^{20}$ & $5.5 \times 10^{19}$ & $5.5 \times 10^{15}$ \\
\hline
\end{tabular}

Table 3. Frequency of messages intercepted at the earth.

${ }^{\mathrm{a}}$ The numerical-example value.

Table 4. Distances to the most distant stars/exoplanets within the $0.1 d$ and $0.001 d$ radius interstellar communication.

\begin{tabular}{ccc}
\hline $\begin{array}{c}d \\
\text { Years between } \mathbf{C} \text { to } \mathbf{D}\end{array}$ & $\begin{array}{c}\text { Distance to the farthest star/exoplanet } \\
\text { for } 0.1 d \text { Light Years }\end{array}$ & $\begin{array}{c}\text { Distance to the farthest } \\
\text { star/exoplanet for 0.001dLight Years }\end{array}$ \\
\hline 4000 & 200 & 2 \\
40,000 & 2000 & 100 \\
200,000 & 10,000 & 1500 \\
300,000 & 15,000 & $\mathbf{2 0 0 0}$ \\
$\mathbf{4 0 0 , 0 0 0}^{\mathbf{a}}$ & $\mathbf{2 0 , 0 0 0}$ & 20,000 \\
$4,000,000$ & 200,000 & 200,000 \\
$40,000,000$ & $2,000,000$ &
\end{tabular}

${ }^{\mathrm{a}}$ The numerical-example value. 
out by these advanced civilizations since we believe curiosity would be a strong motivation among all intelligent civilizations. Communications with and among such groups will also be subject to interception.

In addition, there is no reason to rule out exoplanets hundreds of thousands if not millions of light years away from our local cluster. Consider entities that have much longer lifetimes, are not obliterated in millions of years, and are populated by cyborgs having, for example, a hundred-million-year lifetimes. For such entities a million light-year travel to the environs of planet Earth would be like "an afternoon walk in the park" and their intercommunication range sphere could be billions of light years across! On the other hand, an omnidirectional wave pattern has been assumed whereas transmissions by HFGW transmitters may be projected along narrow beams as discussed in Baker and Baker [5]. Nevertheless, it would appear, on balance that the chances are we would "encounter" intelligent extraterrestrial creatures soon after we fabricated and operated HFGW detectors sensitive enough for eavesdropping or intercepting their messages! However, so far it has been assumed that advanced extraterrestrial civilizations would want to communicate among themselves and with other like civilizations in our Universe. It is believed that intercommunication is an essential feature of all life forms: from Indian smoke signals among tribes to laser inter-satellite communications-even animals intercommunicate for very practical reasons such warning or danger signals or the dancing of bees to communicate paths to nectar. There is somehow a basic need of all creatures to chat and even gossip! Finally, do not be too pessimistic about the lifetimes of extraterrestrial civilizations. Diamandis and Kotler in their book Abundance [46] suggest that "the future is better than you think" and civilizations such as ours may not easily self-destruct.

\section{The Proper Detectors Sensitive Enough to Sense Their Intercommunications}

Mayor and Queloz [47] discovered the first exoplanet in 1995. This discovery opened the floodgates that lead to the amazingly large number of exoplanets, a portion of which could be cradles of intercommunicating advanced intelligent beings. We ponder that, with the identification of this myriad of exoplanets by NASA's Kepler Satellite, even in the limited regional volume of our Universe near our Solar System, are we very near to the cataclysmic event of extraterrestrial interaction? The September 8-15, 2014 edition of TIME Magazine (p. 85) posed the question "How soon will we discover Alien life?" They assumed 100 billion star systems that might support life and that "Estimates of the number of active alien civilizations range from 10,000 to one million." Of course, "discovering alien life" is a far different matter than estimating their existence! One wonders, however, why such "advanced" entities would bother with us today at all? It would highly improbable that intelligent entities would be much interested in us and try to travel or communicate with us-how many times have you attempted to communicate with an anthill? On the other hand, it seems likely to us, and is the basic theme of the discussion, that there would be interstellar intercommunication among intelligent extraterrestrial entities or their "ships" and we might be able to eavesdrop or listen in to their chatter ("gossip"). Since interstellar space is mainly a high vacuum, ordinary acoustic or sound communication would of course be impossible. Such alien entities might utilize microwave radio communication. The "Search for Extraterrestrial Intelligence" or SETI program assumes this communication mode and others believe lasers might be utilized by such entities. More recently, as reported by Ian O’Neil on July $20^{\text {th }}, 2015$

(http://news.discovery.com/space/alien-life-exoplanets/hawking-spearheads-new-hunt-for-intelligent-aliens-150 720.htm) Stephen Hawking announced a the launch of “... a new \$100 million effort, funded by a grant from Yuri Milner, to track down alien civilizations within the next decade ....” As previously stated in the Introduction, it is seriously doubted that such intelligent creatures would choose electromagnetic radiation communication means since such radiation would be so easily attenuated or absorbed by intervening matter such as stars or other interstellar material. In a lecture RMLB delivered in 1961 with Robert Forward, RMLB suggested (Forward and Baker, [48]) that the only appropriate means for interstellar communication (and even local on-planet communications for example, like the trans-Earth HFGW transmitter discussed by Baker and Baker [5]) would be to utilize a radiation that is not so easily absorbed: gravitational radiation or gravitational waves, which like gravity itself passes through most media completely unattenuated. Such radiation was originally proposed in 1905 by Jules Henri Poincaré, a famous French scientist and mathematician, and developed in a quantitative theory by Albert Einstein in 1916. These gravitational waves were indirectly proven to exist by R. A. Hulse and J. H. Taylor, Jr.'s observations of double neutron stars (PSR $1913+16$ ), who were awarded the Nobel Prize for their work. This prompted the development of the Laser Interferometer Gravitational-Wave Observatory or LIGO (Abramovici, [49]) primarily due to the work of Kip S. Thorne at Caltech. Soon the European Space 
Agency's Laser Interferometer Space Antenna (LISA) Pathfinder will start to test gravitational-wave detection technologies for a mission set to launch in 2034. While LIGO and LISA are designed to detect gravitational waves having an extremely low frequency (fractions of a cycle per second), which are theoretically established to be generated by the merger of two black holes, viable interstellar communication would require the use of High-Frequency Gravitational Waves e.g., $100 \mathrm{MHz}$ or higher (in order to provide essential bandwidth like today's "4Gigabyte" smart phones) and cannot be detected by large-scale interferometer devices such as LIGO, LISA, Virgo, GEO 600, et al. (Shawhan, [50]) ${ }^{1}$. are also possible consequences of a rapid inflation of the early Universe; please see for example, Grishchuk [51] and Spergel [52] or, possibly, from supermassive black holes during galaxy mergers (Shannon, et al., [53]).Therefore, it is concluded that to prepare for the eventuality of an intercept of interstellar intercommunication among intelligent extraterrestrial entities, we would be well advised to detect and listen to high-frequency gravitational waves, understand their "form" and also to prepare ourselves mentally and emotionally to "meet" such extraterrestrial entities electronically via such HFGW communications. In Figure 5 are shown gravitational waves compared to other forms of waves. As far as laboratory HFGW generators or transmitters are concerned, a number of them have already been designed (Romero, [54]), Portillaand Lapiedra [55], Grishchuk [56], Kolosnitsyn and Rudenko [57], Baker and Baker, [58] based upon Baker [59] and this double-helix HFGW generator or transmitter (utilizable for through-Earth or trans-global communications) is summarized in Baker and Baker [5]. In particular, if nanotechnology is utilized, then trans-global communication can implemented on Earth. For example, from Brinker and Clem [60] if the Film Bulk Acoustic Re-

\section{WHAT ARE GRAVITATIONAL WAVES?}

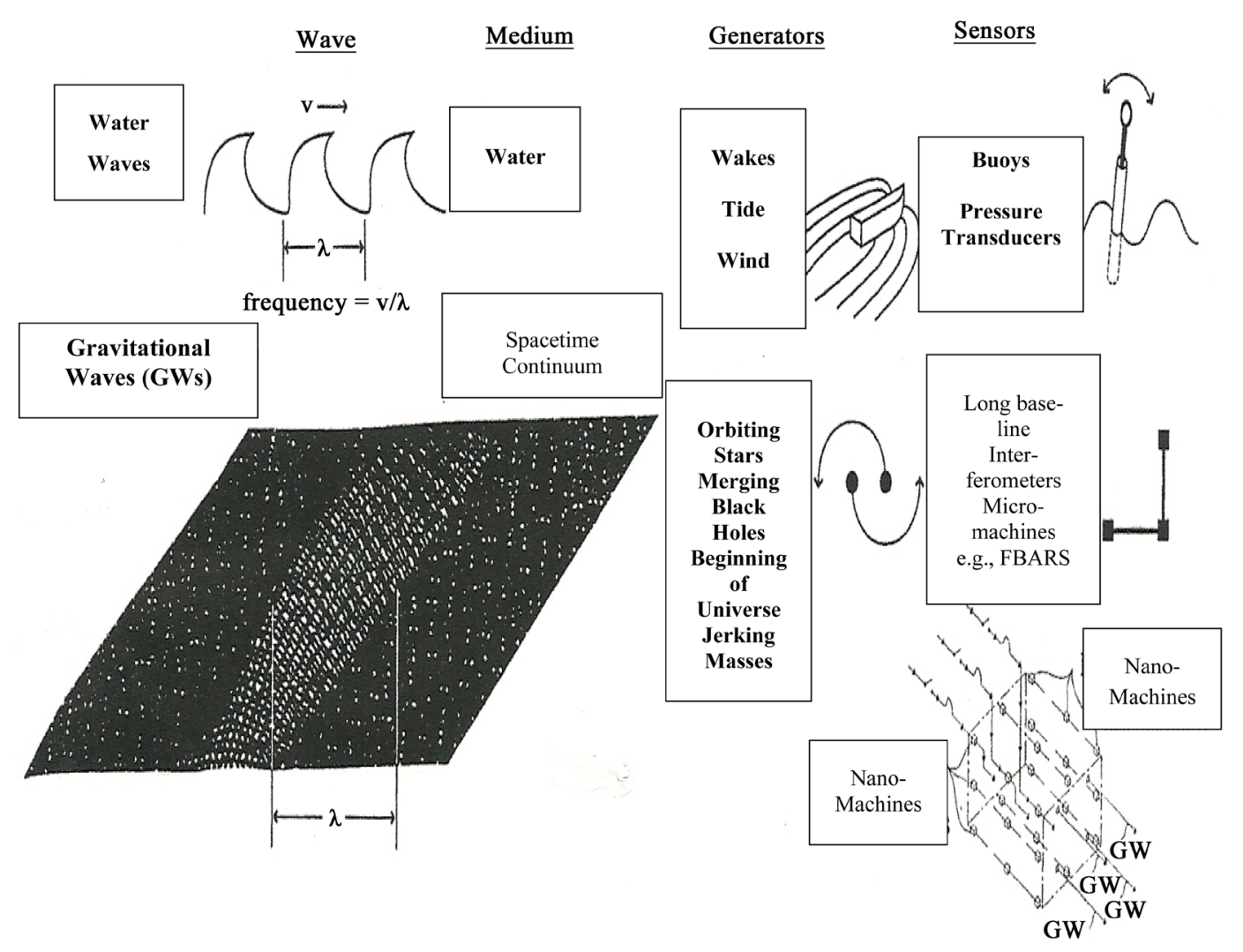

Figure 5. Gravitational waves.

\footnotetext{
1“At higher frequencies (above a kilohertz) the quantum nature of the laser beam (made up of discrete photons, albeit a large number of them) limits the precision of the measurements. Increased laser power would reduce the problem of quantum noise, but ultimately, the LIGO (-like) interferometers are not suited to measuring gravitational waves that stretch or shrink the arms much more rapidly than the time a photon typically remains in the optical cavity (laser arm), which is roughly a millisecond for these (long) interferometers (or approximately a one-kilocycle frequency limit) ... (Shawan, [50], p. 356).
} 
sonators could be reduced from $110 \mu \mathrm{m}$ to less than $11 \mu \mathrm{min}$ size due to the ultra-small quartz crystals, then there would be a 1000 fold increase in HFGW amplitude to $A \approx 10^{-27} \mathrm{~m} / \mathrm{m}$ with a 1500 signal-to-noise ratio one Earth diameter from the HFGW transmitter (Baker and Baker, [5]). Some of the currently designed HFGW detectors could receive that signal! Surely, an advanced exoplanetary civilization would be able to transmit (and receive) HFGW signals with ease both locally (for on-planet communications) and for interstellar communications, it would be the communications medium of choice.

The main concern would be in the interception of interstellar communications. For significant interstellar communications bandwidths HFGW frequencies of $100 \mathrm{MHz}$ or higher would be desirable. In this regard, there are currently (2015) six high-frequency gravitational wave (HFGW) detectors or receivers in operation or under development none of which is similar to the detector found deficient in the well-publicized JASON study (please see http://www.gravwave.com/docs/Q\%20\&\%20A.pdf).One of the first practical HFGW detectors was developed at Birmingham University, England by Mike Cruise and his graduate student Richard Ingley. The Birmingham HFGW detector measures changes in the polarization state of a microwave beam (indicating the presence of a GW) propagating within a waveguide loop about one meter diameter, as shown in Figure 6. It is expected to be sensitive to HFGWs having spacetime fractional deformations or strain of $A \sim 2 \times 10^{-13} / \sqrt{ } \mathrm{Hz}$ meters per meter as reported by Cruise [3] [61]-[63] and Ingley [64] [65].

The INFN Genoa HFGW resonant antenna consists of two coupled, superconducting, spherical, harmonic oscillators a few centimeters in diameter, shown in Figure 7. The oscillators are designed to have (when uncoupled) almost equal resonant frequencies. In theory the system is expected to have a sensitivity to HFGWs with amplitude (fractional deformations or strain) of about $\sim 2 \times 10^{-17} / \sqrt{\mathrm{Hz}}$ meters per meter with an expectation to reach a sensitivity of $\sim 2 \times 10^{-20} / \sqrt{ } \mathrm{Hz}$ (Chincarini and Gemme, [66], Bernard, [67], and Ballantini, [68]).
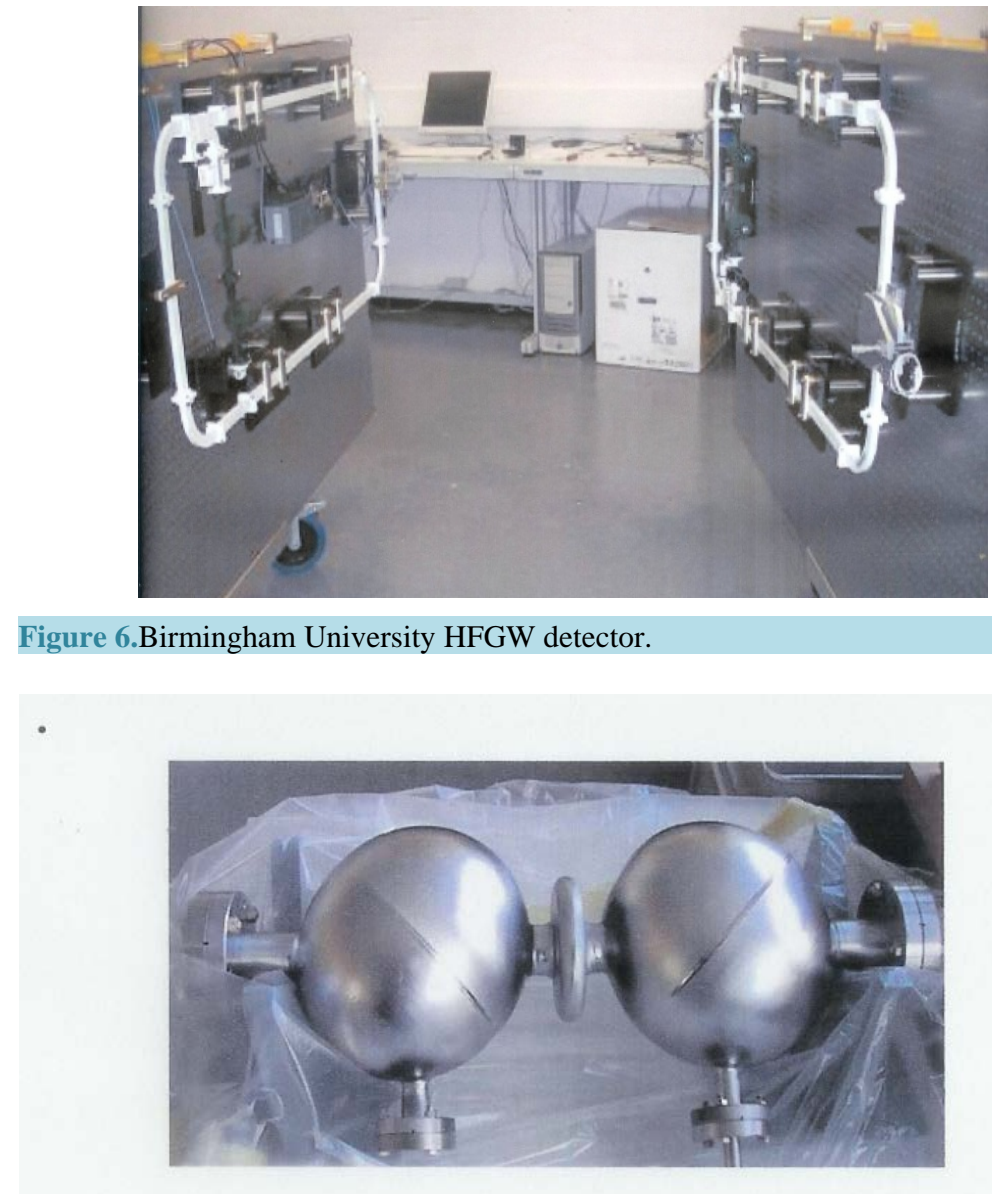

Figure 7. INFN Genoa HFGW detector, photo by author. 
The Kawamura 100 MHz HFGW detector has been built by the Astronomical Observatory of Japan. It consists of two synchronous interferometers with arm lengths of $75 \mathrm{~cm}$ as shown in Figure 8. Its sensitivity is now about $10^{-16} / \sqrt{\mathrm{Hz}}$ meters per meter (Drever and Kowalski, [69] and Nishizawa, et al., [70]). According to Cruise [63], its frequency is limited to $100 \mathrm{MHz}$ and at higher frequencies its sensitivity diminishes. This detector is similar to a miniature LIGO and its schematic is shown in Figure 8.

Arvanitaki, [4] of Stanford University and the University of Nevada, developed a means of detecting HighFrequency Gravitational Waves with Optically Levitated Sensors,” as shown in Figure 9.

In addition, the two most recent and probably much more sensitive High-Frequency Gravitational Wave Detectors under development are shown in Figure 10 and Figure 11. It also should be noted at this point that there may be "optical" devices or HFGW "lenses" that could concentrated the HFGWs at these more sensitive HFGW detectors or receivers using superconductor materials (Woods, [71]). The first of the new HFGW detectors is called the Li-Baker. It is based upon an effect determined by Fangyu Li of Chongqing University, China and Patented in 2001 by R. M L Baker, Jr., (Baker, [72]). The Li effect or Li theory was first published in 1992 [73]. Subsequently, the "Li effect" has been the subject of many peer-reviewed research journal articles (e.g., [74][79]).

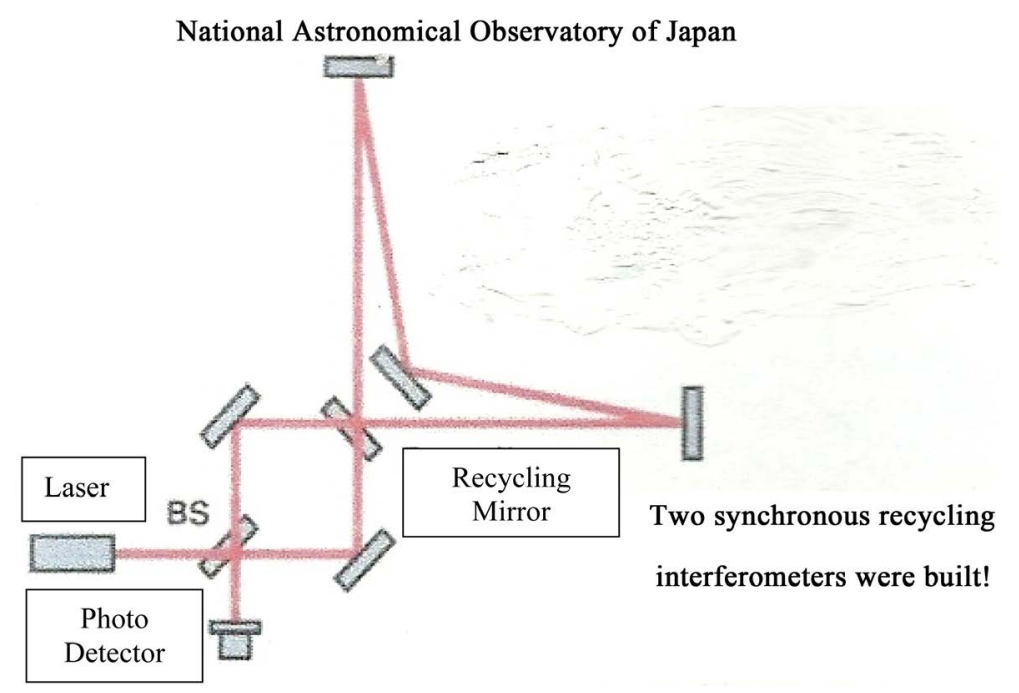

Figure 8. National astronomical observatory of Japan 100 MHz HFGW detector (Nishizawa, et al., [70]).

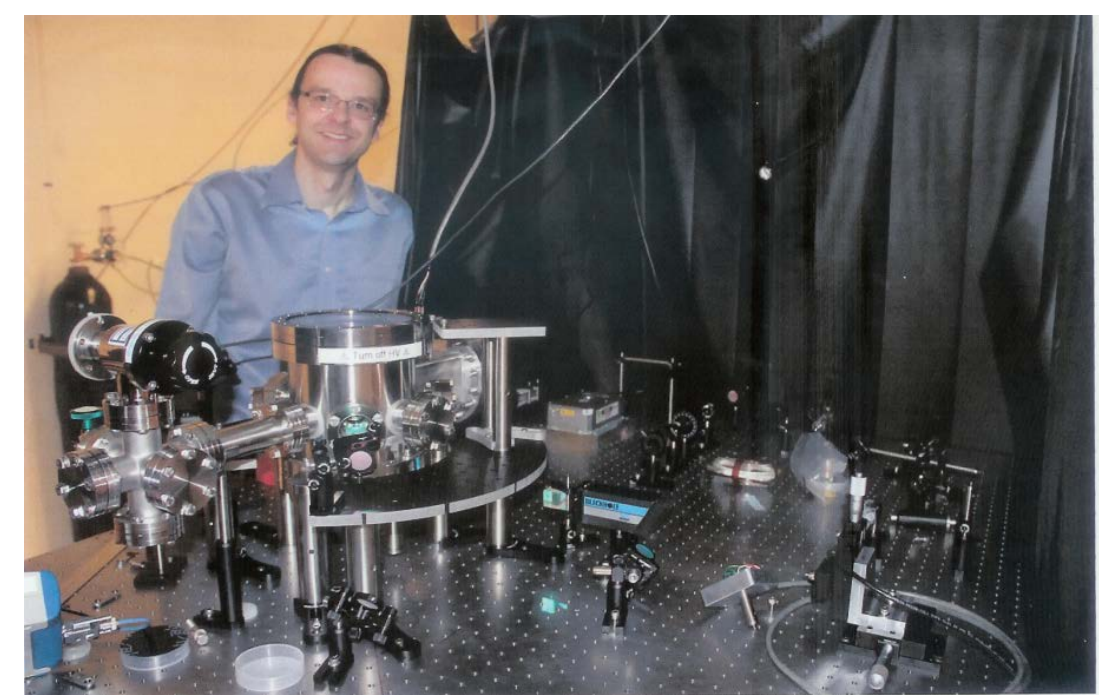

Figure 9. Stanford/university of Nevada HFGW detector 50 to $300 \mathrm{kHz}$ [4]. 


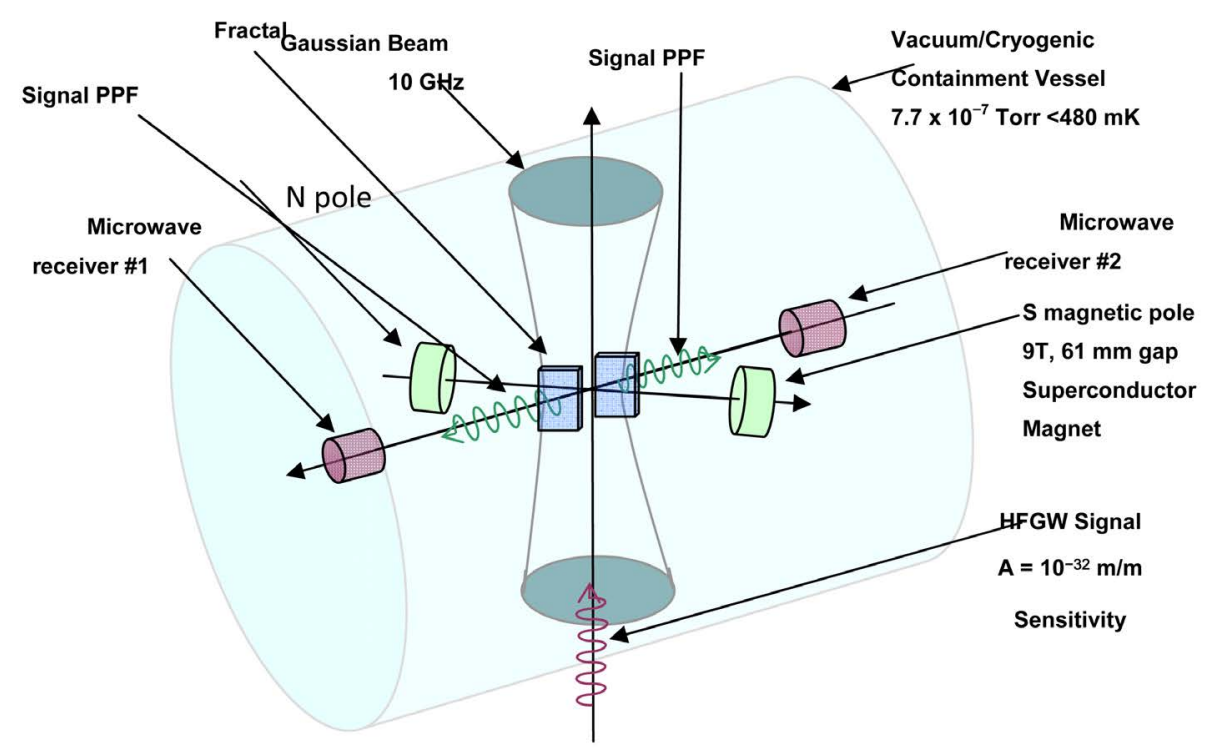

Figure 10. Notional drawing of Li-Baker detector by the author.
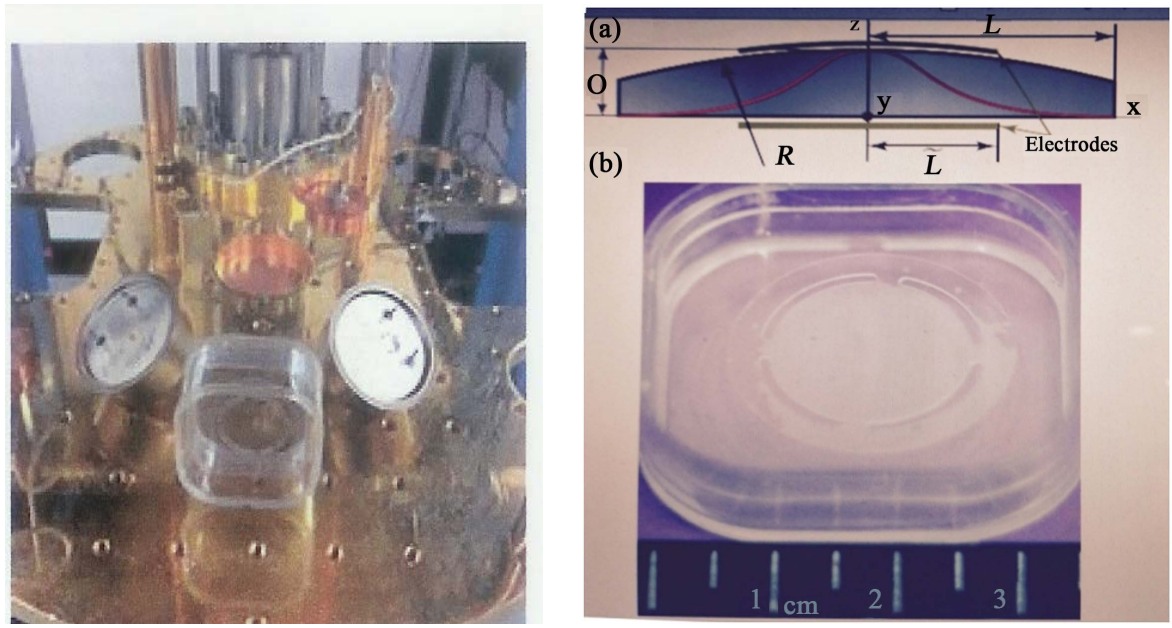

Figure 11. Gravitational wave detection with high frequency phonon trapping acoustic cavities (Goryachev and Tobar [81]).

The key results and formulas are found in Woods, [80] and there is a detailed discussion of the coupling between HFGWs, a magnetic field and a microwave beam in Li [77] [79]. The Li effect is very different from the classical (inverse) Gertsenshtein effect. In the Li effect, a gravitational wave interacts with a laboratory-generated electromagnetic (EM) wave in the presence of a static magnetic field.In the (inverse) Gertsenshtein-effect detectors there is no laboratory-generated electromagnetic wave and the Gertsenshtein effect is extremely small and has no value for HFGW detection.

As of 2015, the Li-Baker detector is under development by Chongqing University in China, High-magneticfield Center of Chinese Academy of Science (construction of high background magnetic field) and Southwest Jiaotong University in China. So far, the Li-Baker would be the most sensitive detector of weak signal highfrequency gravitational waves exhibiting an expected sensitivity of $10^{-32}$ meters per meter amplitude strain in the fabric of spacetime as determined by the analyses of Woods, [80].

Another of the most recent HFGW detectors has been developed by Maxim Goryachev and Michael E. Tobar [81] at the ARC Centre of Excellence for Engineered Quantum Systems, School of Physics, University of Western Australia and is shown in Figure 11. It utilizes High Frequency Phonon Trapping Acoustic Cavities. No sensitivity values have so far (2015) been published. 


\section{Conclusions}

Resulting from the observations of the Kepler satellite observatory, it is predicted that there are many sextillions of exoplanets in our Universe and that some of them may harbor extraterrestrial intelligent entities. If natural selection exists everywhere in the Universe, then such extraterrestrial entities must have those qualities that help ensure permanence. One probable evolving alien life form would involve the replacement or repair of their "parts" especially if they were largely composed of electronic components as we predicted for human life forms. As it has been stated such cyborgs would be essentially immortal or at least very long-lived. Also it is essential to identify and quantify the means of their interpersonal intercommunication. A step in that direction is presented in Hirschberg and Manning [82]. An obvious conclusion is that evolution will produce some form of efficient and unmitigated, yet editable, direct brain-to-brain or mind-to-mind intercommunication. Certainly, the direct "plug" to one brain in Figure 2(a) that transfers thoughts to controla complex prosthetic device, Figure 2(b), could eventually be expected to direct thoughts to and from another brain. Such intercommunication signals would modulate the High-Frequency Gravitational Waves or HFGWs, illustrated in Figure 12. The concept is similar to the modulation of radio waves by sound waves. It would be like an advanced cipher or encrypted message to be decoded and we would be eavesdropping on "them” using High-Frequency Gravitational Wave detectors. In this same regard, Fangyu Li [83] suggested that pulsed HFGWs might be the sign that they were signals from an advanced intelligent civilization. Such impulsive HFGWs also might be a radiative form emitted from cosmic strings (Wen, [84]). It is again emphasized that, unlike electromagnetic radiation, HFGWs are not easily absorbed. Forward and Baker some 55 years ago, suggested the use of HFGWs to monitor extraterrestrial intelligence communications [48] and soon that prediction might come to pass.

It is strongly recommended to prepare for and encourage the cataclysmic event of extraterrestrial interaction in two major ways:

1) Conduct research and development of high-frequency gravitational wave (HFGW) detectors or receivers. There are now (2015) six either fabricated or under development. So far, those HFGW detectors that have actually been built may not have the required sensitivity that would be necessary of interstellar communication interception.

2) Conduct research in cryptography with special attention to the possible interception and decoding of "brain-wave" or Brain-to-Brain modulated HFGW intercommunication from extraterrestrial interstellar and local, on-planet, communication intercepts. This will involve the teaming of neuroscientists and cryptographic experts, the later probably from institutions such as the National Security Agency of the United States and the former from research groups such as the UCLA Brain Research Institute.

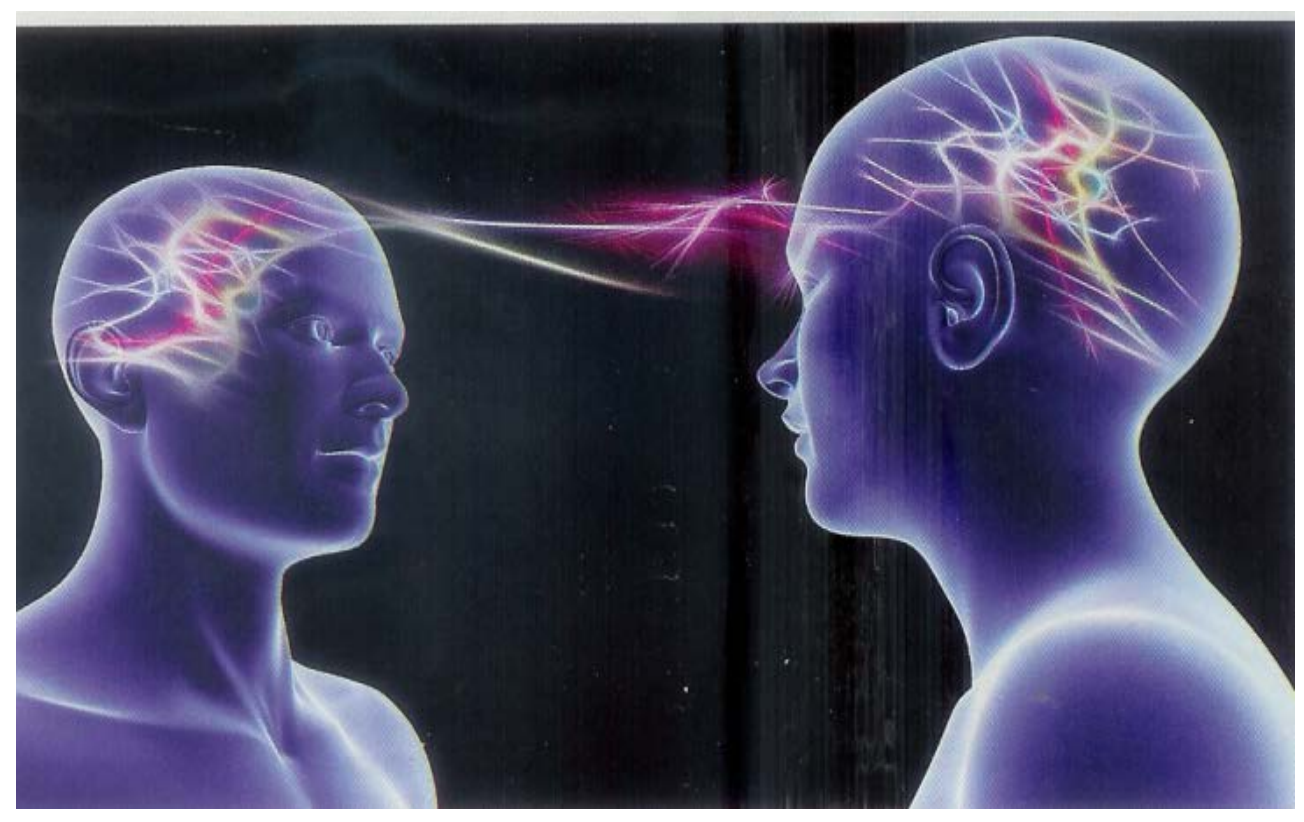

Figure 12. Brain-to-brain direct communication (Power, [85] and Grey [86]). 


\section{Acknowledgements}

Transportation Sciences Corporation has supported the research and preparation of this paper, in part.

\section{References}

[1] Service, R.F. (2013) The Cyborg Era Begins. Science, 340, 1162-1165.

http://dx.doi.org/10.1126/science.340.6137.1162

[2] Veloso, M. (2015) Embrace a Robot-Human World. Nature, 521, 415-418.

[3] Cruise, A.M. (2000) An Electromagnetic Detector for Very-High-Frequency Gravitational Waves. Classical and Quantum Gravity, 17, 2525-2530. http://dx.doi.org/10.1088/0264-9381/17/13/305

[4] Arvanitaki, A.G. and Andrew, A. (2013) Detecting High-Frequency Gravitational Waves with Optically Levitated Sensors. Physical Review Letters, 110, Article ID: 071105. http://dx.doi.org/10.1103/PhysRevLett.110.071105

[5] Baker Jr., R.M.L. and Baker, B.S. (2015) Double-Helix, High-Frequency Gravitational Wave Generator Utilizing Nano Piezoelectric Crystals. Proceedings of 4th International Symposium on Energy Challenges, 11-13 August 2015, Session on Mechanics 07: Nanotechnology for Generators, Aberdeen.

http://www.drrobertbaker.com/docs/Double\%20Helix\%20HFGW\%20Gen.\%20V7.pdf

[6] Abbott, A. (2015) Neuroscience: Drama of Consciousness. Science, 518, 166.

[7] Wilkinson, D. (2013) Science, Religion and the Search for Extraterrestrial Intelligence. Oxford University Press, Oxford. http://dx.doi.org/10.1093/acprof:oso/9780199680207.001.0001

[8] Wade, L. (2015) Birth of the Moralizing Gods. Science, 349, 918-922. http://dx.doi.org/10.1126/science.349.6251.918

[9] Cruz, M. and Coontz, R. (2013) Alien Worlds Galore. Science, 340, 565-581. http://dx.doi.org/10.1126/science.340.6132.565

[10] Brandeker, A. (2014) A Different Class of Planets. Science, 343, 1440-1441. http://dx.doi.org/10.1126/science.1251123

[11] Jontof-Hutter, D., Rowe, J.F., Lissauer, J.J., Fabrycky, D.C. and Ford, E.B. (2015) The Mass of the Mars-Sized Exoplanet Kepler-138 b from Transit Timing. Nature, 522, 321-323. http://dx.doi.org/10.1038/nature14494

[12] Tollefson, J. (2015) Climate Scientists Join Search for Alien Earths. Nature, 520, 420. http://dx.doi.org/10.1038/520420a

[13] Scharf, C. (2015) Whose Galaxy Is It? Columbia University, New York, 36.

[14] Ballard, S., Chaplin, W.J., Charbonneau, D., Désert, J.-M., Fressin, F., Zeng, L., Werner, M.W., Davies, G.R., Aguirre, V.S., Basu, S., Christensen-Dalsgaard, J., Metcalfe, T.S., Stello, D., Bedding, T.R., Campante, T.L., Handberg, R., Karoff, C., Elsworth, Y., Gilliland, R.L., Hekker, S., Huber, D., Kawaler, S.D., Kjeldsen, H., Lund, M.N. and Lundkvist, M. (2014) Kepler-93b: A Terrestrial World Measured to Within 120 km, and a Test Case for a New Spitzer Observing Mode. The Astrophysical Journal, 790, 12. http://dx.doi.org/10.1088/0004-637X/790/1/12

[15] Jenkins, J.M., Twicken, J.D., Batalha, N.M., Caldwell, D.A., Cochran, W.D., Endl, M., Latham, D.W., Esquerdo, G.A., Seader, S., Bieryla, A., Petigura, E., Ciardi, D.R., Marcy, G.W., Isaacson, H., Huber, D., Rowe, J.F., Torres, G., Bryson, S.T., Buchhave, L., Ramirez, I., Wolfgang, A., Li, J., Campbell, J.R., Tenenbaum, P., Sanderfer, D., Henze, C.E., Catanzarite, J.H., Gilliland, R.L. and Borucki, W.J. (2015) Discovery and Validation of Kepler-452b: A 1.6-Earth-Radius Super Earth Exoplanet in the Habitable Zone of a G2 Star. The Astrophysical Journal, 150, 56. http://dx.doi.org/10.1088/0004-6256/150/2/56

[16] Dutrey, A., Di Folco, E., Guilloteau, S., Boehler, Y., Bary, J., Beck, T., Beust, H., Chapillon, E., Gueth, F., Huré, J.-M., Pierens, A., Piétu, V., Simon, M. and Tang, Y.-W. (2014) Possible Planet Formation in the Young, Low-Mass, Multiple Stellar System GG Tau A. Nature, 514, 600-602. http://dx.doi.org/10.1038/nature13822

[17] Quintana, E.V., Barclay, T., Raymond, S.N., Rowe, J.F., Bolmont, E., Caldwell, D.A., Howell, S.B., Kane, S.R., Huber, D., Crepp, J.R., Lissauer, J.J., Ciardi, D.R., Coughlin, J.L., Everett, M.E., Henze, C.E., Horch, E., Isaacson, H., Ford, E.B., Adams, F.C., Still, M., Hunter, R.C., Quarles, B. and Selsis, F. (2014) An Earth-Sized Planet in the Habitable Zone of a Cool Star. Science, 344, 277-280. http://dx.doi.org/10.1126/science.1249403

[18] Witz, A. (2015) Planet Hunters Plot a Course. Nature, 514, 8-9.

[19] Wittenmyer, R.A., Tuomi, M., Butler, R.P., Jones, H.R.A., Anglada-Escudé, G., Horner, J., et al. (2014) GJ 832c: A Super-Earth in the Habitable Zone. The Astrophysical Journal, 791, 114. http://dx.doi.org/10.1088/0004-637x/791/2/114

[20] Bromley, B. and Kenyon, S. (2015) Planet Formation around Binary Stars: Tatooine Made Easy. The Astrophysical Journal, 806, 98. http://dx.doi.org/10.1088/0004-637x/806/1/98 
[21] Deamer, D. (2014) Origin of Life: The First Spark. Nature, 514, 302-303. http://dx.doi.org/10.1038/514302a

[22] Perkins, S. (2014) From Hell on Earth, Life’s Building Blocks. Science, 346, 1279. http://dx.doi.org/10.1126/science.346.6215.1279

[23] Knoll, A.H. (2015) Life on a Young Planet. Princeton University Press, Princeton.

[24] Wolf, S.R. (2015) Science and the Search for Meaning. Science, 347, 137-138. http://dx.doi.org/10.1126/science.aaa0328

[25] Wilson, E.O. (2014) The Meaning of Human Existence. Liveright, New York, 208 p.

[26] Koch, C. (2013) The End of the Beginning for the Brain. Science, 339, 759-760. http://dx.doi.org/10.1126/science.1233813

[27] Kurzweil, R. (2012) How to Create a Mind. Viking Press, New York.

[28] Gershman, S.L.J., Horvitz, E.J. and Tenenbaum, J.B. (2015) Computational Rationality: A Converging Paradigm for Intelligence in Brains, Minds, and Machines. Science, 349, 273-278. http://dx.doi.org/10.1126/science.aac6076

[29] Baker Jr., R.M.L. (2013) About Face. Economic Round Table at The California Club, Los Angeles. http://www.drrobertbaker.com/docs/AboutFace.pdf

[30] Noy, A. (2011) Bionanoelectronics. Advanced Materials, 23, 807-820. http://dx.doi.org/10.1002/adma.201003751

[31] Underwood, E. (2014) AAAS Collections in Neuroscience. Science, 345, 385-386.

[32] Gulati, T., Won, S.J., Ramanathan, D.S., Wong, C.C., Bodepudi, A., Swanson, R.A. and Ganguly, K. (2015) Robust Neuroprosthetic Control from the Stroke Perilesional Cortex. The Journal of Neuroscience, 35, 8653-8661.

[33] Machens, C.K. (2012) Building the Human Brain. Science, 338, 1156-1157. http://dx.doi.org/10.1126/science.1231865

[34] Mannoor, M.S., Jiang, Z., James, T., Kong, Y.L., Malatesta, K.A., Soboyejo, W.O., Verma, N., Gracias, D.H. and McAlpine, M.C. (2013) 3D Printed Bionic Ears. Nano Letters, 13, 2634-2639. http://dx.doi.org/10.1021/nl4007744

[35] Marx, V. (2015) Tissue Engineering: Organs from the Lab. Nature, 522, 373-377. http://dx.doi.org/10.1038/522373a

[36] Ledford, H. (2015) The Printed Organs Coming to a Body near You. Nature, 520, 273. http://dx.doi.org/10.1038/520273a

[37] Liu, D. (2015) DNA-Based Gel for Printing Organs. Nature, 518, 458. http://dx.doi.org/10.1038/518458c

[38] Fritzsch, B. (2014) Electric Organs, History and Potential. Science, 345, 631-632. http://dx.doi.org/10.1126/science.345.6197.631-b

[39] Hansen, M., et al. (2014) Science, Diplomacy and Beyond Electric Organs. Otology \& Neurotology, 34, 1682.

[40] Kazerooni, H. (2008) Exoskeleton for Human Performance Augmentation. In: Siciliano, B. and Khatib, O., Eds., Springer Handbook of Robotics, Springer, Berlin, 773-792. http://202.120.43.103/downloads2/7b95dd28-743a-488f-9e22-68ab47b60dee.pdf http://dx.doi.org/10.1007/978-3-540-30301-5_34

[41] Merolla, P., Arthur, J.V., Alvarez-Icaza, R., Cassidy, A.S., Sawada, J., Akopyan, F., Jackson, B.L., Imam, N., Guo, C., Nakamura, Y., Brezzo, B., Vo, I., Esser, S.K., Appuswamy, R., Taba, B., Amir, A., Flickner, M.D., Risk, W.P., Manohar, R. and Modha, D.S. (2014) A Million Spiking-Neuron Integrated Circuit with a Scalable Communication Network and Interface. Science, 345, 668-673. http://dx.doi.org/10.1126/science.1254642

[42] Reardon, S. (2014) US Regulators Move on Thought-Controlled Prosthetics. Nature, 515, 476. http://dx.doi.org/10.1038/515476a

[43] Pruszynski, J.A. and Diedrichsen, J. (2015) Reading the Mind to Move the Body. Science, 348, 860-861. http://dx.doi.org/10.1126/science.aab3464

[44] Erwin, D.H. (2015) Extinction. Princeton University Press, Princeton.

[45] Silver, M.W. (2004) Ham Radio for Dummies. Wiley Publishing, Indianapolis.

[46] Diamandis, P.H. and Kotler, S. (2012) Abundance. Free Press, New York.

[47] Mayor, M. and Queloz, D. (1995) A Jupiter-Mass Companion to a Solar-Type Star. Nature, 378, 355-359. http://dx.doi.org/10.1038/378355a0

[48] Forward, R.L. and Baker Jr., R.M.L. (1961) Gravitational Gradients, Gravitational Waves and the Weber Bar. Lecture Given at the Lockheed Astrodynamics Research Center, LARC, 650 N. Sepulveda, Bel Air, California, USA, November 16, Lockheed Research Report RL 1521.

[49] Abramovici, A., Althouse, W.E., Drever, R.W.P., Gürsel, Y., Kawamura, S., Raab, F.J., Shoemaker, D., Sievers, L., Spero, R.E., Thorne, K.S., Vogt, R.E., Weiss, R., Whitcomb, S.E. and Zucker, M.E. (1992) LIGO: The Laser Interferometer Gravitational Wave Observatory. Science, 256, 325-333. http://dx.doi.org/10.1126/science.256.5055.325 
[50] Shawhan, P.S. (2004) Gravitational Waves and the Effort to Detect Them. American Scientist, 92, 350-356. http://dx.doi.org/10.1511/2004.48.938

[51] Grishchuk, L.P. (2006) Relic Gravitational Waves and Cosmology. Uspekhi Fizicheskikh Nauk, 176, 1-36.

[52] Spergel, D. and Keating, B. (2015) The Oldest Cosmic Light. Nature, 518, 170-171. http://dx.doi.org/10.1038/518170a

[53] Shannon, R.M., Ravi, V., Lentati, L.T., Lasky, P.D., Hobbs, G., Kerr, M., et al. (2015) Gravitational Waves from Binary Supermassive Black Holes Missing in Pulsar Observations. Science, 349, 1522-1525.

http://dx.doi.org/10.1126/science.aab1910

[54] Romero, B. and Dehnen, H. (1981) Generation of Gravitational Radiation in the Laboratory. Zeitschrift für Naturforschung A, 36, 948-955. http://dx.doi.org/10.1515/zna-1981-0905

[55] Portilla, M. and Lapiedra, R. (2001) Generation of High Frequency Gravitational Waves. Physical Review D, 63, Article ID: 044014. http://dx.doi.org/10.1103/PhysRevD.63.044014

[56] Grishchuk, L.P. (2003) Electromagnetic Generators and Detectors of Gravitational Waves. Gravitational-Wave Conference, The MITRE Corporation, Mclean, 6-9 May 2003, Paper HFGW-03-119.

[57] Kolosnitsyn, N.I. and Rudenko, V. (2007) Generation and Detection of the High Frequency Gravitational Radiation in a Strong Magnetic Field. Proceedings of the HFGW2 Workshop, Institute of Advanced Studies at Austin (IASA), Texas, 19-21 September 2007.

[58] Baker Jr., R.M.L. and Baker, B.S. (2012) Gravitational Wave Generator Apparatus. Proceedings of the Space Technology and Applications International Forum, Albuquerque, 13 March 2012, 288-297.

[59] Baker Jr., R.M.L. (2006) Novel Formulation of the Quadrupole Equation for Potential Stellar Gravitational-Wave Power Estimation. Astronomische Nachrichten/Astronomical Notes, 327, 710-713. http://www.gravwave.com/docs/Astronomische\%20Nachrichten\%202006.pdf http://dx.doi.org/10.1002/asna.200510617

[60] Brinker, J.C. and Clem, P.G. (2013) Quartz on Silicon. Science, 340, 818-819. http://dx.doi.org/10.1126/science.1236752

[61] Cruise, A.M. and Ingley, R.M.L. (2005) A Correlation Detector for Very High Frequency Gravitational Waves. Classical and Quantum Gravity, 22, 5479-5481. http://dx.doi.org/10.1088/0264-9381/22/10/046

[62] Cruise, A.M. (2007) Operational Performance of the Birmingham $100 \mathrm{MHz}$ Detector and Upper Limits on the Stochastic Background. Amaldi 7 Gravitational Wave Conference, Sydney, 9 July 2007.

[63] Cruise, A.M. (2008) Very High Frequency Gravitational Waves. Gravitational Wave Advanced Detector Workshop (GWADW), Elba Conference, 17 May, Slide Presentation 132.

[64] Ingley, R.M.J. and Cruise, A.M. (2001) An Electromagnetic Detector for High Frequency Gravitational Waves. 4th Edoardo Amaldi Conference on Gravitational Waves, Perth, 8-13 July 2001.

[65] Ingley, R.M.J. (2005) Implementation and Cross Correlation of Two High Frequency Gravitational Wave Detectors. PhD Thesis, The University of Birmingham, Birmingham.

[66] Chincarin, A. and Gemme, G. (2003) Micro-Wave Based High-Frequency Gravitational Wave Detector. Gravitational-Wave Conference, The MITRE Corporation, Mclean, 6-9 May 2003, Paper HFGW-03-103.

[67] Bernard, P., Gianluca, G., Parodi, R. and Picasso, E. (2001) A Detector of Small Harmonic Displacements Based on Two Coupled Microwave Cavities. Review of Scientific Instruments, 72, 2428-2437. http://dx.doi.org/10.1063/1.1366636

[68] Ballantini, R., Bernard, P., Calatroni, S., Chiaveri, E., Chincarini, A., Croce, R.P., Cuneo, S., Galdi, V., Gemme, G., Losito, R., Parodi, R., Picasso, E., Pierro, V., Pinto, I.M., Podesta', A. and Vaccarone, R. (2005) Microwave Apparatus for Gravitational Waves Observation. INFN Technical Note INFN/TC-05/05, gr-qc/0502054.

[69] Drever, J.L. and Kowalski, F.V. (1983) Laser Phase and Frequency Stabilization Using an Optical Resonator. Applied Physics B, 31, 97-105. http://dx.doi.org/10.1007/BF00702605

[70] Nishizawa, A., Kawamura, S., Akutsu, T., Arai, K., Yamamoto, K., Tatsumi, D., Nishida, E., Sakagami, M., Chiba, T. Takahashi, R. and Sugiyama, N. (2008) Laser-Interferometric Detectors for Gravitational Wave Backgrounds at 100 Mhz: Detector Design and Sensitivity. Physical Review D, 77, Article ID: 022002. http://dx.doi.org/10.1103/physrevd.77.022002

[71] Woods, R.C. (2007) Modified Design of Novel Variable-Focus Lens for VHFGW. AIP Conference Proceedings, 880, 1011-1018. http://dx.doi.org/10.1063/1.2437545

[72] Baker Jr., R.M.L. (2001) Gravitational Wave Generator. Chinese Patent No. 01814223.0. http://www.gravwave.com/docs/Chinese\%20Detector\%20Patent\%2020081027.pdf

[73] Li, F., Tang, M. and Zhao, P. (1992) Interaction between Narrow Wave Beam-Type High Frequency Gravitational 
Radiation and Electromagnetic Fields. Acta Physica Sinica, 41, 1919-1928

[74] Li, F. and Tang, M. (1997) Positive Definite Problem of Energy Density and Radiative Energy Flux for Pulse Cylindrical Gravitational Wave. Acta Physica Sinica, 6, 321-333.

[75] Li, F., Tang, M., Luo, J. and Li, Y.-C. (2000) Electrodynamical Response of a High-Energy Photon Flux to a Gravitational Wave. Physical Review D, 62, Article ID: 044018. http://dx.doi.org/10.1103/PhysRevD.62.044018

[76] Li, F., Tang, M. and Shi, D.-P. (2003) Electromagnetic Response of a Gaussian Beam to High-Frequency Relic Gravitational Waves in Quintessential Inflationary Models. Physical Review D, 67, Article ID: 104008. http://dx.doi.org/10.1103/PhysRevD.67.104008

[77] Li, F., Yang, N., Fang, Z., Baker Jr., R.M.L., Stephenson, G.V. and Wen, H. (2009) Signal Photon Flux and Background Noise in a Coupling Electromagnetic Detecting System for High-Frequency Gravitational Waves. Physical Review D, 80, Article ID: 064013.

http://www.gravwave.com/docs/Li,\%20et\%20al.\%20July\%202009,\%20HFGW\%20Detector\%20Phys.\%20Rev.\%20D. pdf http://dx.doi.org/10.1103/PhysRevD.80.064013

[78] Li, F. and Baker Jr., R.M.L. (2007) Detection of High-Frequency Gravitational Waves by Superconductors. International Journal of Modern Physics B, 21, 3274-3278. http://dx.doi.org/10.1142/S0217979207044366

[79] Li, F., Baker Jr., R.M.L., Fang, Z., Stephenson, G.V. and Chen, Z. (2008) Perturbative Photon Fluxes Generated by High-Frequency Gravitational Waves and Their Physical Effects. The European Physical Journal C, 56, 407-423. http://www.drrobertbaker.com/docs/Li-Baker\%206-22-08.pdf http://dx.doi.org/10.1140/epjc/s10052-008-0656-9

[80] Woods, R.C., Baker Jr., R.M.L., Li, F., Stephenson, G.V., Davis, E.W. and Beckwith, A.W. (2011) A New Theoretical Technique for the Measurement of High-Frequency Relic Gravitational Waves. Journal of Modern Physics, 2, 498-518. http://vixra.org/abs/1010.0062 http://www.gravwave.com/docs/J.\%20of\%20Mod.\%20Phys\%202011.pdf http://dx.doi.org/10.4236/jmp.2011.26060

[81] Goryachev, M. and Tobar, M.E. (2014) Gravitational Wave Detection with High Frequency Phonon Trapping Acoustic Cavities. Physical Review D, 90, Article ID: 102005. http://dx.doi.org/10.1103/PhysRevD.90.102005

[82] Hirschberg, J. and Manning, C.D. (2015) Advances in Natural Language Processing. Science, 349, 261-266. http://dx.doi.org/10.1126/science.aaa8685

[83] Li, F. (2015) Private Communication, 10 November 2015.

[84] Wen, H., Li, F., Fang, Z. and Beckwith, A. (2014) Impulsive Cylindrical Gravitational Wave: One Possible Radiative Form Emitted from Cosmic Strings and Corresponding Electromagnetic Response. European Physical Journal C, 74, 2998. http://dx.doi.org/10.1140/epjc/s10052-014-2998-9

[85] Power, S.A. (2014) Where I End, Where You Begin. Science, 345, 262. http://dx.doi.org/10.1126/science.1255866

[86] Grey, R. (2016) Could We Soon “Speak” Telepathically? Mind-Reading Computer Deciphers Words from Brainwaves BEFORE They Are Spoken. Daily Mail. http://www.dailymail.co.uk/sciencetech/article-3386875/Could-soon-speak-telepathically-Mind-reading-computer-deci phers-words-brainwaves-spoken.html\#ixzz3wbHFNGQS 